\title{
El tratamiento médico fue más efectivo que la angioplastia en reducir el riesgo de muerte e infarto en pacientes con angina
}

Coronary angioplasty versus medical therapy for angina: the second Randomised Intervention Treatment of Angina ( RITA-2 ).

Rita-2 trial participants. Lancet 1997; 350: 461-68

\author{
Objetivo \\ Comparar la angioplastia (AP) vs. el tratamiento medico (TM) como \\ estrategia inicial en pacientes con enfermedad coronaria y compro- \\ miso leve a moderado.
}

\section{Diseño}

Ensayo clínico randomizado, multicéntrico. Seguimiento: 2,7 años.

\section{Lugar}

20 centros cardiológicos en el Reino Unido e Irlanda

\section{Pacientes}

Se incluyeron 1018 pacientes con enfermedad coronaria comprobada por arteriografía; a los que un cardiólogo de referencia consideró a la angioplastia o el tratamiento médico como alternativas similares. Se excluyeron pacientes con revascularización previa o compromiso del tronco de la coronaria izquierda. El grado de angina así como la cantidad de vasos comprometidos, no fue criterio de exclusión.

\section{Intervención}

Se randomizaron 514 pacientes a TM y 504 a AP en uno o más vasos. El médico tratante podía indicar los procedimientos de revascula rización o los cambios en el tratamiento médico que considerara adecuados, independientemente del grupo asignado.

\section{Medición de resultados principales}

El objetivo primario del estudio fue comparar la mortalidad total e infarto agudo de miocardio (IAM) no fatal (resultado combinado). También se midió la utilización de procedimientos de revascula rización (cirugía y angioplastia) y medicación antianginosa. Se evaluaron los cambios en la clase funcional de la angina.

\section{Resultados}

Durante el seguimiento realizado hubo 32 muertes o IAM no fatales en $\operatorname{AP}(6.3 \%)$ y 17 en $\mathrm{TM}(3.3 \%)$. El riesgo relativo de muerte o IAM no fatal para AP fue 1,96 ( IC $95 \% 1.08-3.41, p=0.02$ ). La diferencia de riesgo absoluto fue $3 \%$ (IC $95 \% 0.4-5.7 \%$ ). No hubo diferencias significativas en la necesidad de cirugía de revascularización: 40 (7.9\%) AP; y $30(5.8 \%)$ TM. Se observó mejoría de la angina en ambos grupos, pero ésta fue mayor en el grupo AP. A los tres meses el grupo TM presentó un $16.5 \%$ más de pacientes con angina en clase funcional $\geq$ II $(p<0.001)$, pero esta diferencia disminuyó a un $7.6 \%$ a los dos años ( $p=0.02)$. La diferencia fue mayor en aquellos pacientes con peor clase funcional inicial. TM utilizó más medicación a lo largo del estudio.

\section{Conclusiones}

La AP comparada con el tratamiento médico (a 2.7 años de seguimiento) aumentó levemente la mortalidad o el riesgo de IAM no fatal en pacientes con compromiso coronario leve o moderado. A su vez la angioplastia mejoró la clase funcional de la angina de pecho en todos los pacientes, pero especialmente en aquellos con síntomas iniciales más severos; esta diferencia se atenuó a los dos años.

\section{COMENTARIO}

Los procedimientos de revascularización han demostrado mejorar los síntomas y reducir la mortalidad en pacientes con angina limitante y pobre respuesta al tratamiento médico y en aquellos con lesiones coronarias particulares (la cirugía demostró ser efectiva en pacientes con enfermedad del tronco de la coronaria izquierda, tres vasos o dos vasos con compromiso de la descendente anterior proximal) (1). En los pacientes con un riesgo menor el tratamiento médico representa una alternativa que brinda similares resultados (2). No había hasta ahora estudios lo suficientemente grandes para comparar y evaluar mortalidad o infarto con ambos métodos. El RITA 2 contesta ese inte rrogante, si bien los resultados definitivos se verán en el seguimiento a cinco años. Sólo la mitad de los pacientes elegibles ingresó al estudio, esto puede explicarse por la preferencia de los médicos por las terapéuticas intervencionistas. A pesar de esto la muestra conseguida es, por sus características, muy representativa de la práctica clínica habitual.

El estudio mostró peores resultados con la AP que con el TM. En base a la diferencia de riesgo observada (3\% [0.4 - 5.7]), por cada 34 pacientes [IC95\% 17 - 250] tratados con AP se produciría una muerte o IAM no fatal (número necesario para dañar*). La necesidad posterior de cirugía o angioplastia fue similar en los dos grupos lo que pone de manifiesto la efectividad del tratamiento médico. Los autores asignan una gran trascendencia a la disminución de la angina en el grupo con angioplastia, pero ésta fue disminuyendo en el seguimiento posterior a 2 años. Además no se debe considerar la angina en forma aislada sino también el impacto de la misma en la calidad de vida de los pacientes. Siendo la enfermedad coronaria un proceso dinámico, la pregunta no debería ser qué tratamiento es mejor, sino qué secuencia de tratamientos y qué combinación resultan más adecuadas para el paciente en cada situación clínica (3). Por último, siempre vale la pena recordar que cuando el riesgo del paciente coronario no es elevado, la disminución de los factores de riesgo son el pilar del tratamiento, ya que se obtienen beneficios superiores al de cualquier procedimiento de revascularización (4). 